\title{
A Novel Quantification Method for Determining Previously Undetected Silent Infarcts on MR-perfusion in Patients Following Carotid Endarterectomy
}

\author{
Xin Liu ${ }^{1}$, Celina Imielinska ${ }^{1,2}$, Joel Rosiene ${ }^{3}$, Anita Rampersad ${ }^{4}$, Joseph Zurica $^{4}$, \\ David Wilson ${ }^{5}$, Hadi Halazun ${ }^{4}$, Susan C. Williams ${ }^{5}$, Angela Ligneli ${ }^{6}$, Anthony \\ D'Ambrosio $^{5}$, Michael Sughrue ${ }^{5}$, E. Sander Connolly ${ }^{5}$, Jr., Eric J. Heyer ${ }^{4}$ \\ ${ }^{1}$ Department of Biomedical Informatics, Columbia University, New York, NY \\ ${ }^{2}$ Department of Computer Science, Columbia University, New York, NY \\ ${ }^{3}$ Department of Mathematics and Computer Science, Eastern Connecticut State University, CT \\ ${ }^{4}$ Department of Anesthesiology, Columbia University, New York, NY \\ ${ }^{5}$ Department of Neurological Surgery, Columbia University, New York, NY \\ ${ }^{6}$ Department of Radiology, Columbia University, New York, NY
}

\begin{abstract}
1. ABSTRACT
The purpose of this paper is to evaluate the post-operative Magnetic Resonance Perfusion (MRP) scans of patients undergoing carotid endarterectomy (CEA), using a novel image-analysis algorithm, to determine if post-operative neurocognitive decline is associated with cerebral blood flow changes. CEA procedure reduces the risk of stroke in appropriately selected patients with significant carotid artery stenosis. However, $25 \%$ of patients experience subtle cognitive deficits after CEA compared to a control group. It was hypothesized that abnormalities in cerebral blood flow (CBF) are responsible for these cognitive deficits. A novel algorithm for analyzing MRperfusion (MRP) scans to identify and quantify the amount of CBF asymmetry in each hemisphere was developed and to quantify the degree of relative difference between three corresponding vascular regions in the ipsilateral and contralateral hemispheres, the Relative Difference Map (RDM). Patients undergoing CEA and spine surgery (controls) were examined preoperatively, and one day postoperatively with a battery of neuropsychometric (NPM) tests, and labeled "injured" patients with significant cognitive deficits, and "normal" if they demonstrated no decline in neurocognitive function. There are apparently significant RDM differences with MRP scans between the two hemispheres in patients with cognitive deficits which can be used to guide expert reviews of the imagery. The proposed methodology aids in the analysis of MRP parameters in patients with cognitive impairment.
\end{abstract}

Keywords: computer-aided diagnosis, magnetic resonance, neurological

\section{INTRODUCTION}

\subsection{Background.}

Stroke is the third leading cause of death in developed countries today. In the United States, over 750,000 patients per year suffer from stroke. Aside from the high degree of mortality associated with cerebral ischemia, strokes may cause tremendous and permanent disability in survivors. 
Strokes are heterogeneous in nature, with several mechanisms potentially leading to cerebral ischemia. In approximately $15-20 \%$ of stroke patients, carotid atherosclerosis is identified as the major stroke risk factor. While few treatments for stroke exist, carotid endarterectomy (CEA) is an effective method of reducing the risk of future stroke in appropriately selected patients with carotid artery stenosis [1-4]. The major complication of this surgical procedure is perioperative stroke. While major stroke is a rare complication, recent work has demonstrated that up to $28 \%$ of patients undergoing CEA experience subtle but clinically detectable neurocognitive deficits following the procedure [5]. Such deficits have been demonstrated by deterioration in performance on a standardized battery of neuropsychometric (NP) tests administered before the operation, and 1 day following the procedure [5]. These neurocognitive changes are believed to be due to ischemic events that occur during the course of CEA. Neurocognitive decline has been associated with elevated serum levels of S100b, a glial cell marker, demonstrating the occurrence of cell death [6]. Potential mechanisms include transient hypoperfusion due to poor collateral circulation or dysfunctional cerebral autoregulation and/or microemboli dislodged during the operation.

\subsection{Purpose}

The purpose of this study is to evaluate the post-operative Magnetic Resonance Perfusion (MRP) scans of patients undergoing CEA, using a novel image-analysis algorithm to identify and quantify the amount of cerebral blood flow (CBF) asymmetry in each hemisphere, and to determine if postoperative neurocognitive decline is associated with cerebral blood flow changes. The proposed algorithm for analyzing post-processed MRP images that quantifies the degree of relative difference between corresponding regions in the ipsilateral and contralateral hemispheres converts CBF values that are meaningless outside of the context of a given scan, to relative "difference", which highlights side-to-side asymmetry. A Relative Difference Map (RDM) [1] was constructed by comparing a small region of the scan to the corresponding region in the contralateral hemisphere, quantifying the degree of relative difference, and representing this quantity of relative difference in 2D and 3D. The amount of relative difference in both hemispheres and in 6 major vascular territories was analyzed to assess the degree of hypoperfusion in the regions. An approach that automatically identifies the asymmetry and quantifies the amount of asymmetry has been proposed and implemented. The preliminary results have shown promise in the discovery of pathologic area of MR perfusion that is invisible to human experts or highly variable among different experts. The method automatically identifies the focal points and anatomical regions (vascular territories); derives the axis of reflexional symmetry in each slice in the volumetric MRP-CBF data; computes the RDM; and generates histograms is each vascular territory.

\section{THE METHODOLOGY}

\subsection{Subjects}

Twenty-two patients undergoing elective CEA and two control patients undergoing spine surgery were recruited to participate in this institutional review board-approved study. After written consent was obtained, patients were assessed with a battery of NP tests at two time points; before 
surgery and one day after surgery. Postoperative MRP scans were performed within 24 hours of surgery on all patients.

\subsection{Anesthesia}

No patients were premedicated. All patients received general anesthesia, with routine hemodynamic and temperature monitoring as described previously [5], as well as a radial arterial catheter for measuring blood pressure continuously and an 8-channel EEG (Neurotrac II; Moberg Medical, Inc, Ambler, Pa). Sedation before induction consisted of fentanyl and midazolam. Shunting across the surgical site was performed selectively only if there was evidence of cerebral ischemia by EEG upon clamping the carotid artery. This was defined as a $50 \%$ or greater decrease in amplitude in the alpha or beta frequencies and a similar increase in the delta or theta frequencies. No patients were shunted.

\subsection{Surgery:}

Members of either the neurovascular service or the vascular service performed all CEAs. Surgery consisted of positioning the patient supine with the head in an extended midline position. An incision was made along a skin crease from just below the angle of the mandible to near the midline through skin, subcutaneous tissue, and platysma. The common, internal, and external carotid arteries were exposed and controlled. All patients undergoing CEA received either 5000 or $6000 \mathrm{U}$ of heparin as a bolus. All patients were extubated in the operating room and stayed in an intensive care environment overnight. All patients remained in the hospital for 1 to 3 days for postoperative pain scoring and NP testing.

\subsection{Neuropsychometric Evaluation:}

Patients were assessed with a battery of NP tests. The same research assistant, trained to administer and score these NP tests under the supervision of a neuropsychologist, administered all examinations. Seven raw scores were generated from the battery of NP tests, which were chosen to represent a limited range of cognitive domains. The Boston Naming test was administered to assess verbal fluency and visual identification. Halstead-Reitan Trails parts A and B evaluated visual conceptual and visuomotor tracking by timing how long it took a subject to connect consecutively numbered circles with a single line (part A) and then connect the same number of consecutively numbered and lettered circles by alternating between the two sequences (part B). The Controlled Oral Word Association Test evaluated verbal fluency and provided information on the function of the dominant hemisphere ("left"). Patients were asked to generate as many words as they could, beginning with 3 target letters $(\mathrm{C}, \mathrm{F}$, and $\mathrm{L})$ in 1 minute. Their raw score was the sum of the words produced for all 3 letters. A different set of target letters was provided at follow-up. The Copy Portion of the Rey Complex Figure test was administered. to assess perceptual and visuospatial organization and provides information on the function of the nondominant hemisphere ("right"). Patients were asked to copy the Rey Complex Figure to the best of their ability [7].

To detect cognitive changes, each of the preoperative and postoperative test components was individually scored. The change in each test score was calculated, and was then scaled to test scores of a frequency matched control group of spine patients in order to generate z-scores. The z-scores for each test at each time point were then summed to give a total score for cognitive change. A patient was said to have significant cognitive decline if his or her total score was $\geq 2$ standard deviations from the mean of the total scores of patients in the control group [5]. 


\subsection{Premises and Assumptions.}

For our analysis, we made the following assumptions: (1) In normal cases, the axial MRP images of the left and right hemispheres are, for the most part, structurally symmetric and comparable, and there should be no more than minor side-to-side differences in relative blood flow between the two hemispheres. (2) In abnormal cases, the left and right hemispheres are still structurally symmetric and comparable; but there is significant relative blood flow difference between the two hemispheres, that can be detected using MRP images.

\subsection{Relative Difference Map (RDM)}

A novel algorithm was developed that quantified the degree of relative difference between corresponding regions in the ipsilateral and contralateral hemispheres. A Relative Difference Map (RDM) [9] representing this quantity in 2D and 3D was constructed. The amount of relative difference in both hemispheres and in 6 major vascular territories: left anterior cerebral artery (LACA), left middle cerebral artery (LMCA), left posterior cerebral artery (LPCA), right anterior cerebral artery (RACA), right middle cerebral artery (RMCA), right posterior cerebral artery (RPCA), was analyzed.

A novel approach that automatically identifies the asymmetry and quantifies the amount of asymmetry has been proposed and implemented. Our preliminary results have shown promise in the discovery of pathologic area of MR perfusion that is invisible to human experts or highly variable among different experts. The method automatically identifies the focal points and anatomical regions (vascular territories); derives the axis of reflexional symmetry; computes the RDM; and generates histograms is each vascular territory. The histogram analysis links the quantified symmetry to an assessment of pathology.

The method consists of following steps:

1) From the input image, Fig.1 (a) the center point (centroid) of the anatomical region of interest, is automatically computed, Fig.1 (b);

2) From the centroid, the Fourier Shape Descriptor (FDS) is computed using ray casting [10], where all the pixels inside the brain are parameterized by the angle from the major axis and distance away from the centroid (polar coordinates), Fig 1.(b)

3) The axis of reflection symmetry is derived, , Fig.1 (c).

4) Detect the orientation of the mass by computing the angle between major axis and the vertical coordinate, and re-orientate the brain into the upright position, if needed.

5) Unwrap and re-coordinate the rotated image based on the parameters formulated in step (2). Compute the RDM using 9 by 9 window difference calculation and only highlight the blood deficiency area into the corresponding brain side, Fig 1.(d).

6) Sum up and average out the unwrapped RDMs across the slices, which generates the mean relative difference map that reflects the volumetric information of given scans

7) Divide the unwrapped RDM into 6 vascular territories by roughly estimating the angles, Fig.1 (d). Then disjoin the map Fig.1. (e) and compute the histograms in all six territories, Fig.1 (f).

8) The histogram of the calculated RDM, is examined to quantify the asymmetry 


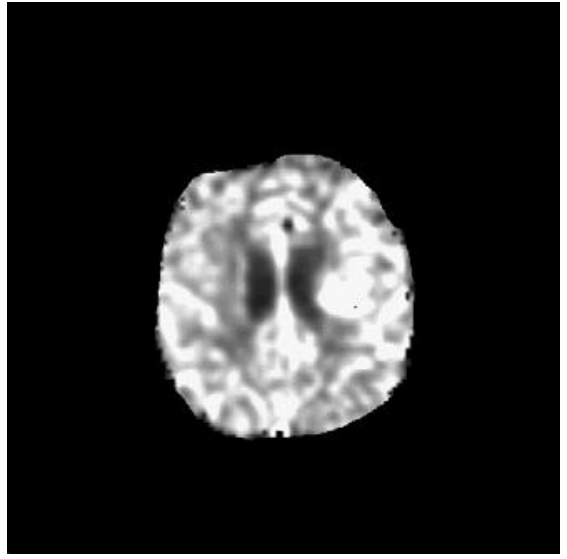

(a)

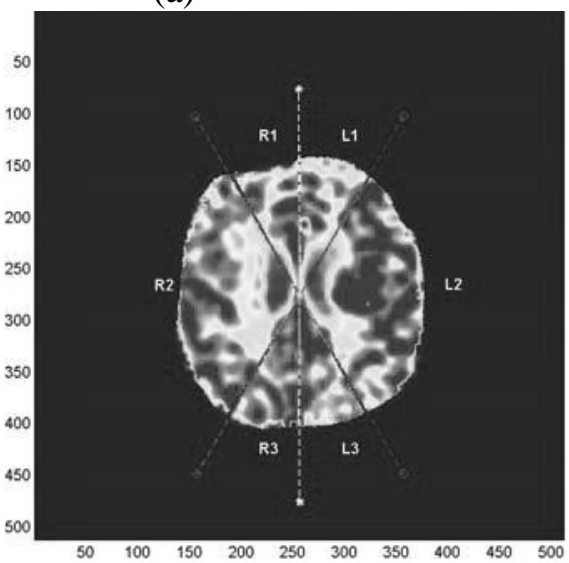

(c)

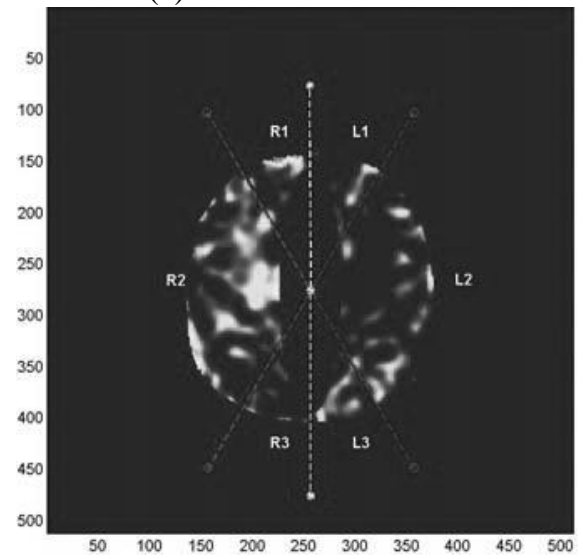

(e)

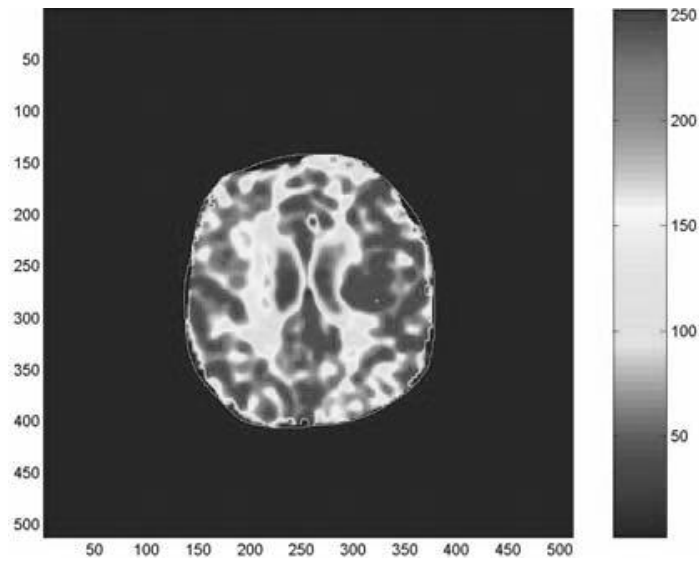

(b)
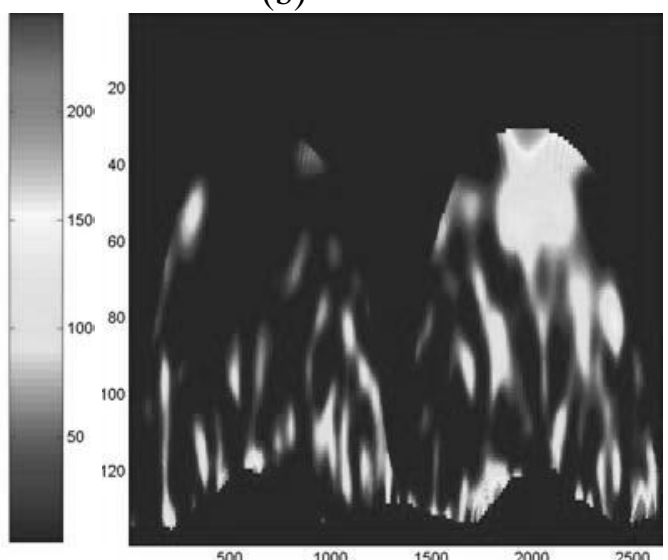

(d)

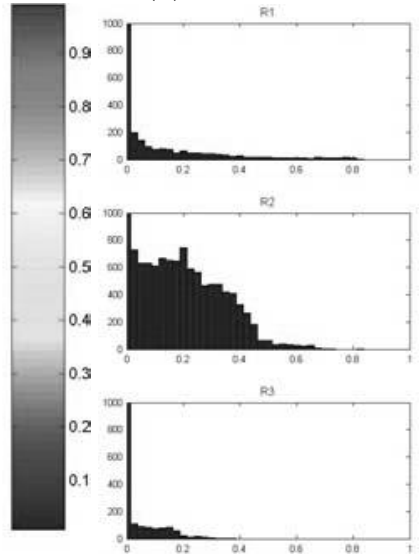

(f)
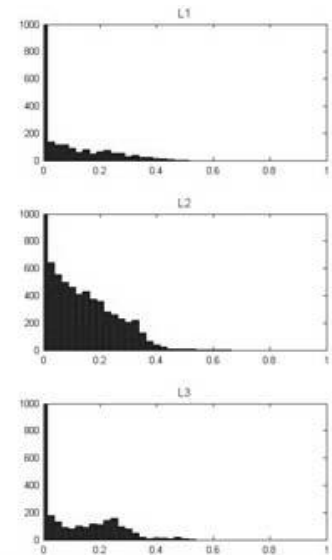

)

Fig.1. Steps of the RDM method: (a) input image, (b) the centroid and Fourier Shape Descriptor (FDS), (c) axis of reflectional symmetry of the brain, (d) unwrapped 2D RDM image from the FDS, (e) RDM with automatically computed six vascular territories: : L1- Left anterior cerebral artery, L2- Left Middle Cerebral artery, L3- Left Posterior cerebral artery, R1- Right, (f) histograms corresponding to the six vascular territories. 
The resulting RDM that is subdivided automatically into the 6 major cerebrovascular territories, and regional histograms are calculated for each territory. Fig.2 represents a hypothetical distribution of histograms of regions in the RDMs computed for CBF. These curves represent the conceptual basis behind our algorithm. We hypothesize that in disease states, "difference" will cluster in affected territories, and will skew the regional histogram to the right in "problem areas."

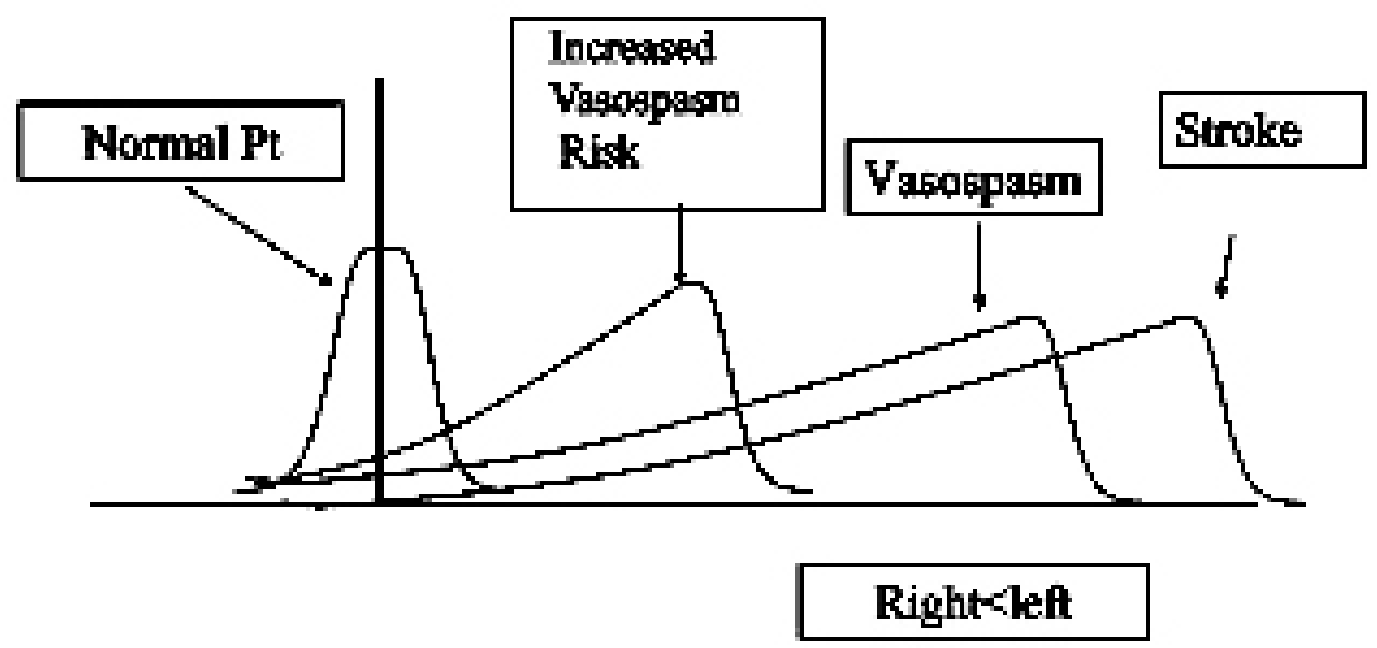

Fig.2. Hypothesized regional histograms quantified from relative difference maps (RDM) of normal patients; and patients with increased vasospasm risk; angiographic vasospasm; and ischemic stroke.

\section{RESULTS AND DISCUSSION}

To illustrate our results, we present two cases, one normal and one "injured" patient, processed by our method. We show a single slice in the MRP-CBF volume.

\subsection{Normal case, Fig.3.}

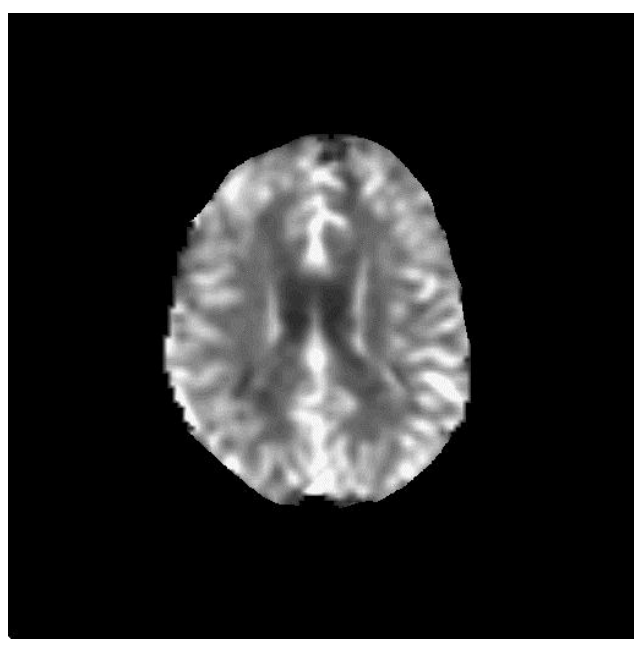

(a)

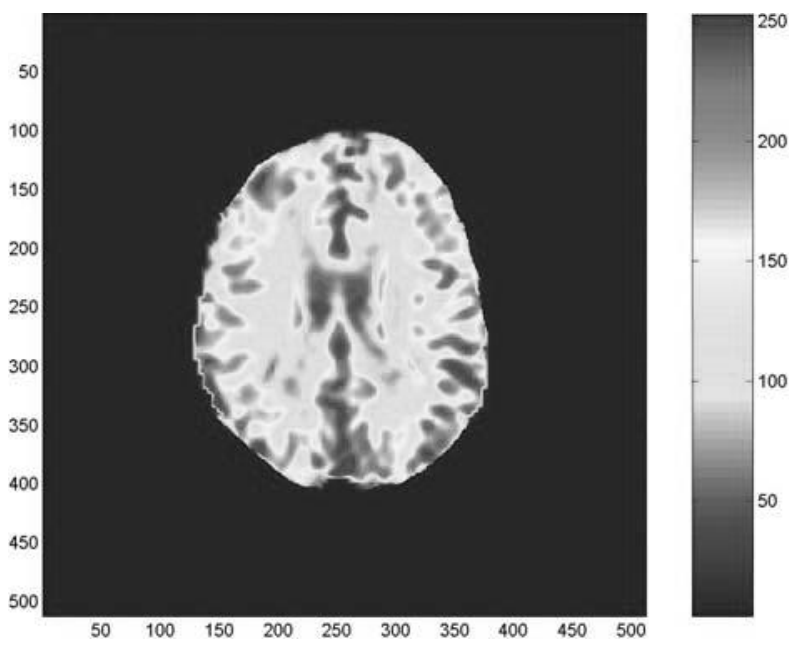

(b) 


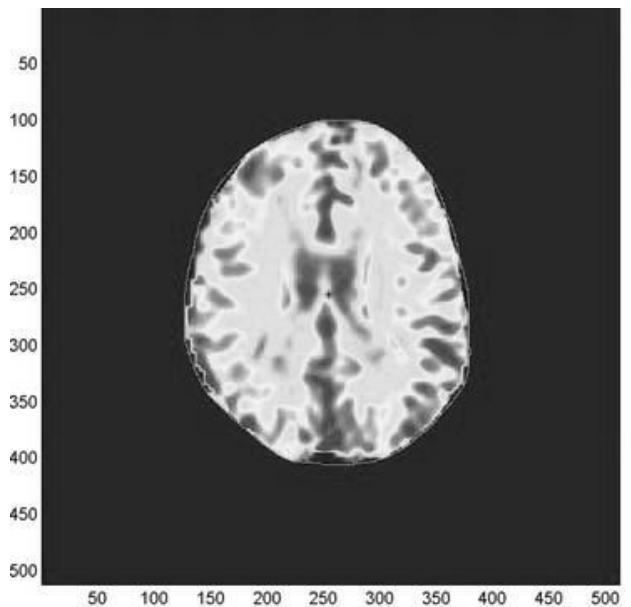

(c)

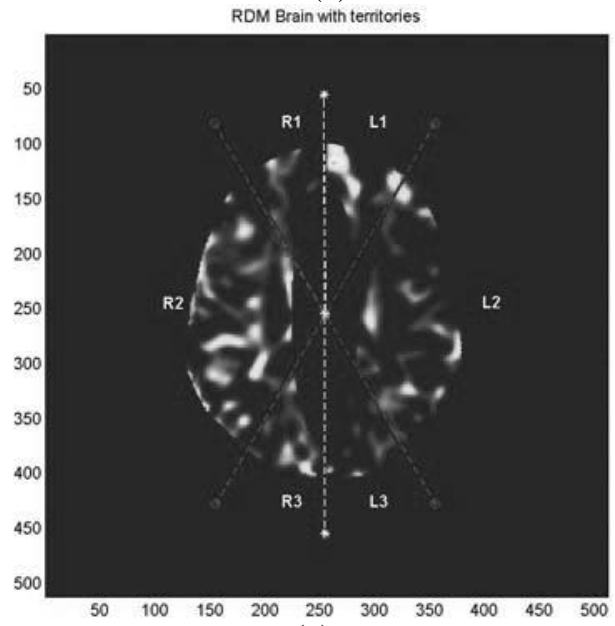

(e)
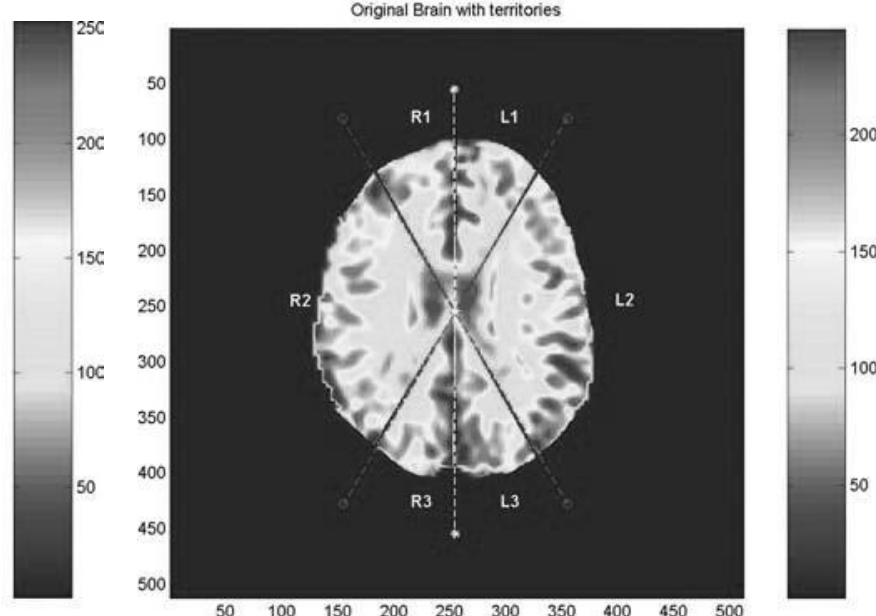

(d)

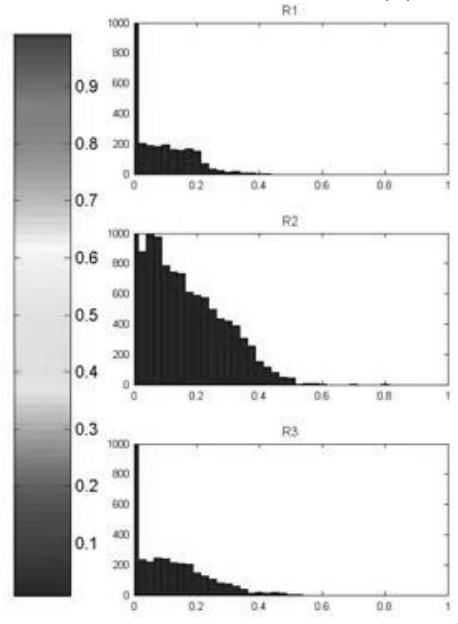

(f)
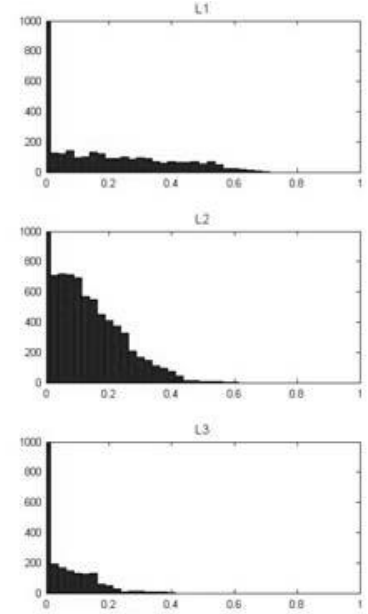

Fig 3. Normal patient; Input MR CBF slice in (a) gray scale intensities, (b) color scale,; (c) "blob"the Fourier Shape Descriptor (FDS) of the brain region; (d) ) the color-mapped images (right) with auto-detected central axis and auto-identified cerebrovascular territories,; (e) RDM - each pixel is represented as a value between 0 and 1 , where higher value depicts the higher difference between left and right hemispheres. (f) Comparison of the RDM histograms of each cerebrovascular territory in each hemisphere (in Fig 2(e)) (left column corresponds to right hemisphere vascular territories, and the right column to the left hemisphere vascular territories) for a patient without significant neuropsychometric changes after CEA. Each territory is relatively symmetrical between the two hemispheres.

\section{2 "Injured" case, Fig.4:}




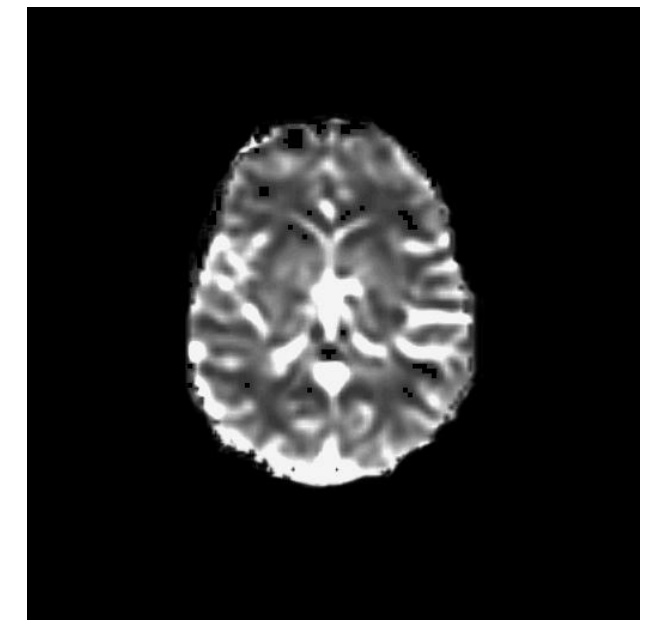

(a)

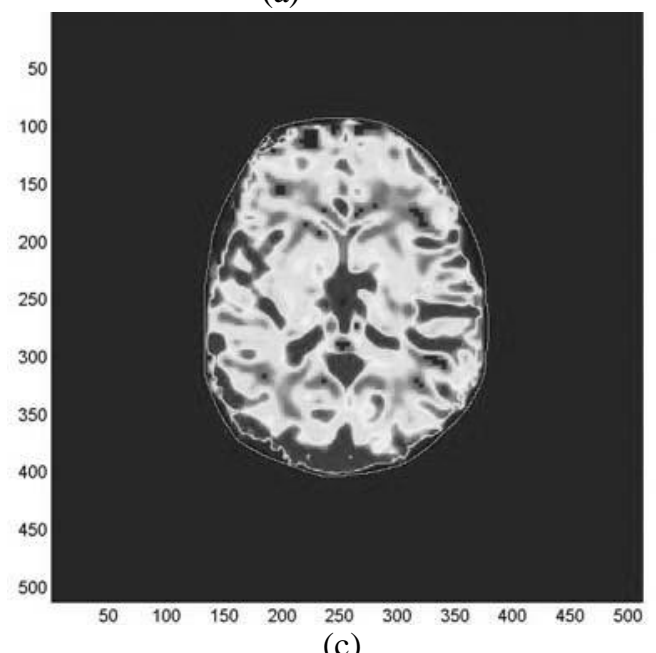

RDM Brain with territories

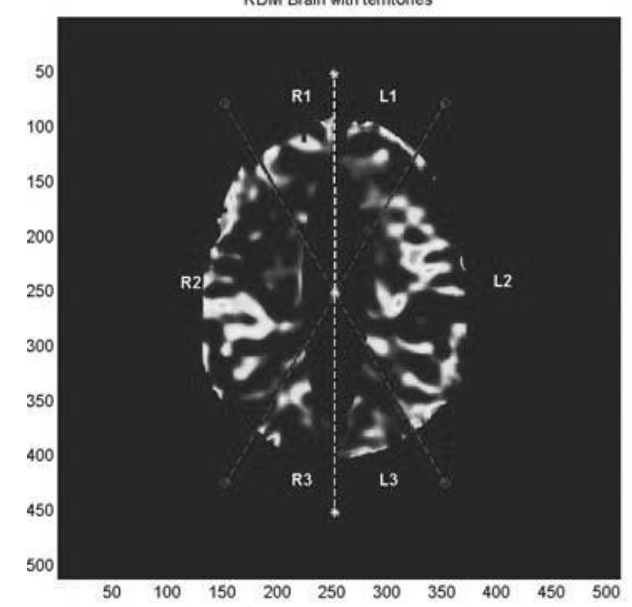

(e)

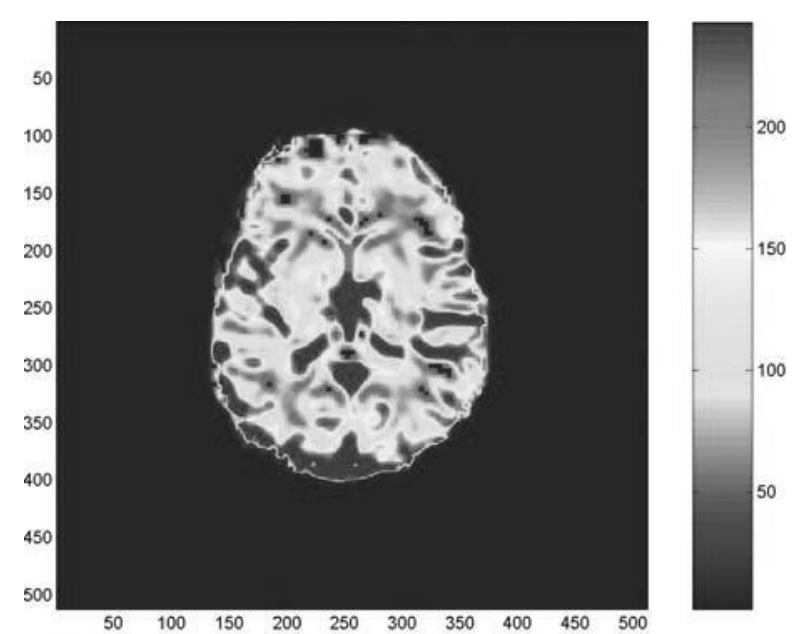

(b)

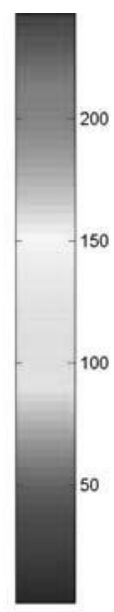

Original Brain with territories

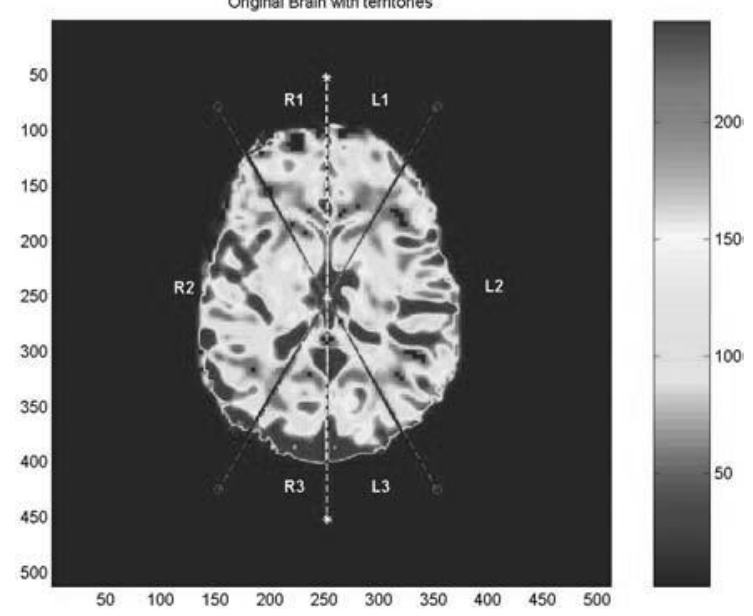

(d)
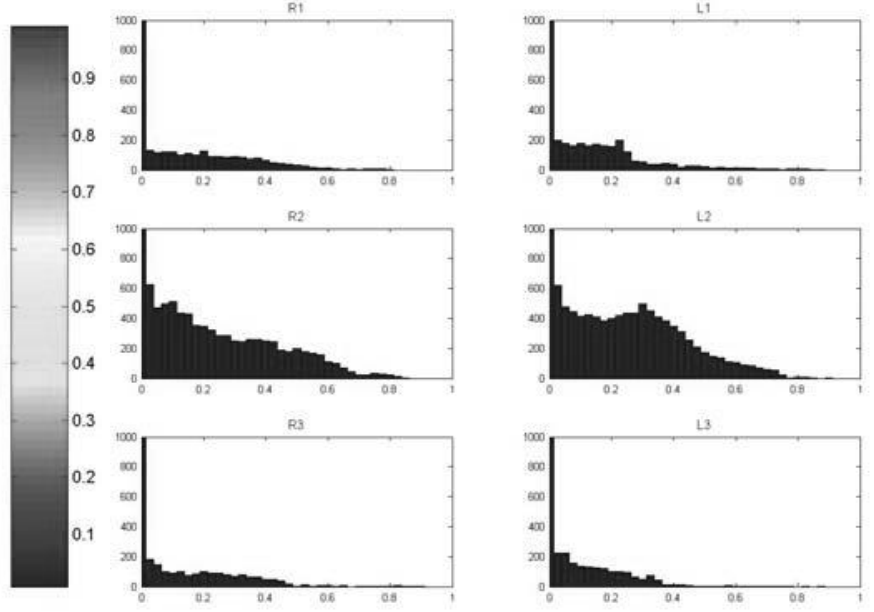

(f)

Fig 4. "Injured" patient with a problem in the left Middle Cerebral Artery (MCA); Input MR CBF slice in (a) gray scale intensities, (b) color scale,; (c) "blob"- the Fourier Shape Descriptor (FDS) of the brain region; (d) the color-mapped images (right) with auto-detected central axis and auto-identified cerebrovascular territories,; (e) RDM - each pixel is represented as a value between 0 and 1, where higher value depicts the 
higher difference between left and right hemispheres. (f) The RDM histogram of each cerebrovascular territory shows a discrepancy between left and right hemispheres (left column corresponds to right hemisphere vascular territories, and the right column to the left hemisphere vascular territories). L2 territory of the Left MCA is relatively asymmetrical, corresponding to an injury in the region.

\subsection{Discussion}

24 MR perfusion scans were of adequate quality to include in our analysis. These scans represent 22 CEAs and 2 spine control cases. Of the 22 CEAs performed, neurocognitive decline was demonstrated on post operative day 1 in 6 cases, yielding an neurocognitive "injury" rate of $27 \%$. This incidence of neurocognitive decline is consistent with prior studies.[5]

All of the 6 patients $(100 \%)$ with detectable postoperative declines in neuropsychometric performance displayed asymmetric blood-flow changes on postoperative MRP scans when we applied out novel algorithm. Our analysis revealed normal symmetric blood flow patterns in the 2 spine control patients and in 11 of 16 (69\%) CEA patients who did not demonstrate neurocognitive decline. The remaining 5 "uninjured" CEA patients displayed asymmetric blood flow patterns, similar to those observed in the "injured" group. These 5 cases represent inconsistencies in our algorithm. However, three of them can be explained by other factors. Two inconsistent cases are explained by asymmetric bifrontal artifarct, likely caused by magnetic interference by dental work. In both cases, the regions displaying blood flow asymmetry correspond to the location of the asymmetric artifact. A third inconsistent case is explained by the occurrence of a pre-operative TIA. This TIA generated a DWI positive lesion in the superior-posterior left frontal lobe within 24hours of the operation. The DWI positive region corresponds to the location of blood flow asymmetry detected by our algorithm. Although this patient did not demonstrate decrease neuropsychometric performance, the agreement between the DWI scan and our findings validates our technique. The final two inconsistent case cannot be explained by asymmetric artifact or cerebral ischemia. Further analysis of the processed MRP scans in these case demonstrates that, when multiple slices are taken into consideration, blood flow appeared more globally symmetric that the result obtained from applying our algorithm to a single slice. When excluding MR perfusion scans with explained inconsistencies, 11 of 13 (85\%) "uninjured" CEA patients displayed symmetric blood flow patterns.

The strong correlation between blood flow asymmetric, as detected by our method, and subtle declines in post-operative cerebral function, suggests that our algorithm is more sensitive than traditional evaluation of MR perfusion scans in detecting blood flow changes. This finding also suggests that hemodynamic alterations may play a role in decreased neuropsychometric performance following CEA.

\section{CONCLUSIONS}

Conventional assessment of MRP scans was unable to demonstrate significant postoperative changes even in patients with postoperative cognitive deficits, whereas asymmetrical changes in MRP were detected by RDMs in patients who had evidence of cognitive deficits. We showed significant RDM differences with MRP scans between the two hemispheres only in patients who had significant cognitive deficits. Our methodology provides a better analysis of MRP parameters in patients with significant cognitive deficits. 


\section{REFERENCE}

1. Collaborators NSCT, Collaborators) NASCET. Beneficial effect of carotid endarterectomy in symptomatic patients with high-grade carotid stenosis. NEJM. 1991;325:445-453

2. Group ECSTC. Mrc european carotid surgery trial: Interim results for symptomatic patients with severe (70-99\%) or with mild (0-29\%) carotid stenosis. Lancet. 1991;337:221-227

3. Hobson II RW, Weiss DG, Fields WS, Goldstone J, Moore WS, Towne JB, Wright CB, Group TVACS. Efficacy of carotid endarterectomy for asymptomatic carotid stenosis. NEJM. 1993;328:221-227

4. Study ECftACA. Endarterectomy for asymptomatic carotid artery stenosis. Executive committee for the asymptomatic carotid atherosclerosis study. Jama. 1995;273:1421-1428

5. Heyer EJ, Sharma R, Rampersad A, Winfree CJ, Mack WJ, Solomon RA, Todd GJ, McCormick PC, McMurtry JG, Quest DO, Stern Y, Lazar RM, Connolly ES. A controlled prospective study of neuropsychological dysfunction following carotid endarterectomy. Arch Neurol. 2002;59:217-222

6. Connolly E, Winfree C, Rampersad A, Sharma R, Mack W, Mocco J, Solomon R, Todd G, Quest D, Stern Y, Heyer E. Serum s100b protein levels are correlated with subclinical neurocognitive declines after carotid endarterectomy. Neurosurgery. 2001;49:1076-1083

7. Lezak MD. Neuropsychological assessment. New York, Oxford: Oxford University Press; 1983.

8. Meyers J, Meyers K. Rey complex figure test and recognition trial professional manual. Odessa, FL: Psychological Assessment Resources, Inc.; 1995.

9. Imielinska C., X. Liu, M. Sughrue, E. Hagiwara, E.S. Connolly, A. D’ Ambrosio, "Objective Quantification of Perfusion-Weighted Computer Tomography in the Setting of Acute Aneurysmal Subarachnoid Hemorrhage", Computer Assisted Radiology and Surgery, pp. 34-43, June 2004.

10. C. Imielinska, X. Liu, J. Rosiene, M. Sughrue, R.J. Komotar, J. Mocco, E.R. Ransom, A. Lignelli, B. E. Zacharia, E.S. Connoly, A. D'Ambrosio, "Towards Objective Quantification of Perfusion-Weighted Computed Tomography in the Setting of Subarachnoid Hemorrhage: Quantification of Symmetry and Automated Delineation of Vascular Territories", accepted to Journal of Academic Radiology Special Issue on Medical Imaging, 2005. 